\title{
Toxoplasma gondii dense granule protein 15 induces apoptosis in choriocarcinoma JEG-3 cells through endoplasmic reticulum stress
}

Wei Wei ${ }^{1}$, Fangfang Zhang ${ }^{2}$, He Chen ${ }^{3}$, Yuanyuan Tang ${ }^{2}$, Tian Xing ${ }^{4}$, Qingli Luo ${ }^{2}$ Li Yu ${ }^{2}$, Jian Du², Jilong Shen ${ }^{1,2,3^{*}}$ and Linjie Zhang ${ }^{1 *}$

\begin{abstract}
Background: Toxoplasma gondii, a single-celled parasite commonly found in mammals, has been shown to induce trophoblast cell apoptosis and subsequently cause fetal damage and abortion. Although dense granule protein 15 (GRA15) has been identified as a key component in innate immunity to T. gondii infection and its pathogenesis, its role in host cell apoptosis remains unclarified.

Methods: Type II GRA15 (GRA15॥) CDNA was inserted into a plasmid encoding enhanced green fluorescent protein (pEGFP). Choriocarcinoma JEG-3 cells were transfected with either pEGFP or pEGFP-GRA15 „॥ and cultured for $24 \mathrm{~h}$. Cell apoptosis and endoplasmic reticulum stress (ERS) responses were assessed. Inhibitors targeting inositol-

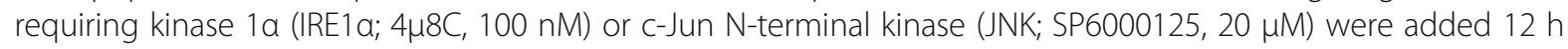
after plasmid transfection, followed by testing the effect of GRA15 „ on ERS.

Results: When compared to pEGFP, pEGFP-GRA15 „transfection facilitated cell apoptosis $(P<0.05)$, increased mRNA expression of caspase-3, caspase-4, 78-kDa glucose-regulated protein (GRP78), C/EBP homologous protein (CHOP) and X-box binding protein-1 (XBP1) (all $P<0.05$ ), and promoted protein expression of cleaved caspase-3, cleaved poly(ADP-ribose) polymerase, Bax, CHOP, GRP78, phospho-JNK, and phospho-IRE1a (all $P<0.05$ ). The $4 \mu 8 \mathrm{C}$ and SP6000125 decreased apoptosis and protein expression of XBP1s, CHOP, TNF receptor-associated factor 2 (TRAF2), phosphorylated apoptosis signal-regulating kinase 1 (ASK1), cleaved caspase-3, phospho-JNK, and Bax (all $P<0.05$ ) in pEGFP-GRA15 |l transfected cells.
\end{abstract}

Conclusions: Toxoplasma GRA15 || induced ERS and subsequently caused apoptosis of choriocarcinoma JEG-3 cells.

Keywords: Toxoplasma gondii, GRA15, Apoptosis, ERS, JEG-3 cell

\section{Background}

Toxoplasma gondii is an obligate intracellular singlecelled parasite that can invade all warm-blooded animals worldwide [1]. The strains of $T$. gondii circulating in Europe and North America can be grouped into three distinct genotypes, strains of Type I, Type II and Type III, according to the population structure [2-5]. During invasion, proteins from parasite organelles such as

\footnotetext{
*Correspondence: shenjilong53@126.com; zlj33@ahmu.edu.cn 'Department of Immunology, School of Basic Medicine, Anhui Medical University, Hefei 230032, China

Full list of author information is available at the end of the article
}

rhoptry proteins (ROPs) and dense granule proteins (GRAs) are released into host cells and are able to cause significant host damage [6, 7]. The genotype/strain polymorphism of ROP16 and GRA15 have both been widely observed in the literature [8]. It has been reported that ROP16 from type I RH stain (ROP16 $)$, but not from type II ME49 stain (ROP16 II), could directly phosphorylate the signal transducer and activator of transcription STAT3 and STAT6, and subsequently polarize macrophages to an M2 phenotype. In addition, GRA15 from type II ME49 strain (GRA15 II), but not from type I RH strain (GRA15 $)$, could phosphorylate nuclear factor-

(c) The Author(s). 2018 Open Access This article is distributed under the terms of the Creative Commons Attribution 4.0 International License (http://creativecommons.org/licenses/by/4.0/), which permits unrestricted use, distribution, and 
Table 1 Sequences of oligonucleotide primers used for real-time PCR

\begin{tabular}{llll}
\hline Target & Forward primer $\left(5^{\prime}\right.$-3') & Reverse primer (5'-3') & Product size (bp) \\
\hline Caspase 3 & GACAGACAGTGGTGTGATG & TGGATGAACCAGGAGCCATC & 132 \\
Caspase 8 & AACCTGGTACATCCAGTCAC & AAAGTAGGCTGAGGCATCTG & 150 \\
Caspase 9 & AAGGTTTAGGACCTTCGAC & GACTGCAGGTCTTCAGAGTG & 184 \\
Caspase 4 & GTGGAGAAGGACTTCATTGC & CTGGAAGCATGTGATGAGTTG & 108 \\
ATF4 & CAGCTACCACCCATAACAAG & GTGTCCATCACCTGACAGTC & 138 \\
ATF6 & CAGCTACCACCCATAACAAG & GTGTCCATCACCTGACAGTC & 133 \\
GRP78 & GTCCTTCTATGAAGGAGAAG & GAATCTTCCAACACTTCTGG & 117 \\
CHOP & TGCAAGAGGTCCTGTCTTCAG & GCACTGACTCCTCGGAAC & 106 \\
XBP1 & AAGGCGCTGAGGAGGAAC & GGTTCTCAACTACAAGGCC & 178 \\
GAPDH & CTTCATTGACCTCAACTACATGG & CTCGCTCCTGGAAGATGGTGAT & 134
\end{tabular}

Abbreviations: ATF activating transcription factor, GRP78 78-kDa glucose-regulated protein, CHOP C/EBP homologous protein, XBP1 X-box binding protein-1, GAPDH glyceraldehyde-3-phosphate dehydrogenase

kappa B (NF-kB), and subsequently drive macrophages to an M1 phenotype [9]. We have previously shown that both ROP16 I and GRA15 1 I were present in the majority of T. gondii Chinese 1 strains found in China [10-14].

Toxoplasma gondii can hijack host cell apoptotic machinery and promote either an anti- or pro-apoptotic program depending on the parasite virulence and load, as well as the host cell type [15]. In the literature, increased apoptosis following Toxoplasma infection has been observed in spleen cells [16], neuronal cells [17] and choriocarcinoma cells [18]. Previously, we found that endoplasmic reticulum stress (ERS) is involved in T. gondii-induced apoptosis [19, 20], and that ROPs could trigger ERS-mediated apoptosis $[21,22]$. However, the effect of GRAs (e.g. GRA15) on host cell apoptosis remains unclear.
Importantly, maternal Toxoplasma infection may give rise to congenital transmission of the parasite to the fetus through the placenta [23-26] and/or via interfering with the immune tolerance on maternal-fetal interface. Our previous studies indicated that infection with $\mathrm{TgCwh} 3$ (a virulent strain of Chinese 1) induced apoptosis of trophoblast cells, and subsequently caused adverse pregnancy outcomes in mice [27]. Angeloni et al. [18] observed that ME49 (type II)-infected BeWo cells become more susceptible to apoptosis than RH (type I)-infected BeWo cells. In view of the M1 bias induced by GRA15 1 , we postulated that a GRA15 ${ }_{\mathrm{II}}$-induced NF-kB-dependent proinflammatory cytokine profile is more likely to cause cell apoptosis when compared to a ROP16 ${ }_{\text {I }}$-induced STAT3/STAT6dependent proinflammatory cytokines $[18,28]$. Here, we

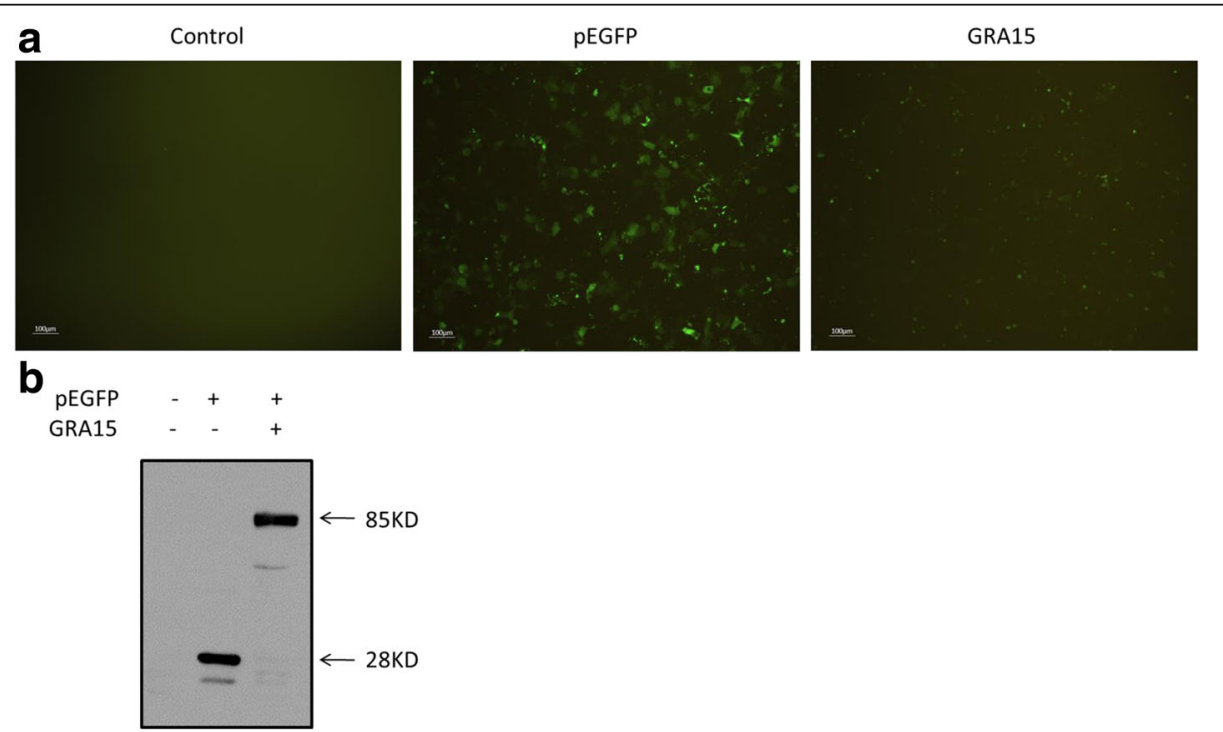

Fig. 1 The presence of dense granule protein 15 (GRA15॥). Choriocarcinoma JEG-3 cells were transfected with either an empty vector (pEGFP, encoding enhanced green fluorescent protein) or pEGFP-GRA15 || for $24 \mathrm{~h}$. Untransfected cells served as the control. a The expression of green fluorescent protein (GFP) was captured using fluorescent microscopy. Scale-bar: $100 \mu \mathrm{m}$. b The expression of GRA15 „was confirmed by western blotting 
demonstrated that GRA15 1 increased apoptosis in choriocarcinoma JEG-3 cells partially mediated by ERS.

\section{Methods}

\section{Cell culture}

JEG-3 cells (human choriocarcinoma cell line, ATCC, lot number HTB-36) were cultured in a humidified incubator $\left(37{ }^{\circ} \mathrm{C}\right.$ and $\left.5 \% \mathrm{CO}_{2}\right)$ in minimum essential medium (MEM; Gibco, Carlsbad, CA, USA), supplemented with sodium bicarbonate (1.5 g/l; Gibco), sodium pyruvate ( 0 . $11 \mathrm{~g} / \mathrm{l}$; Gibco), penicillin (100 U/ml; Sigma-Aldrich, St Louis, MO, USA), streptomycin (100 mg/ml; SigmaAldrich) and fetal bovine serum (10\%; Gibco).

\section{Plasmid construction and transfection}

A plasmid encoding enhanced green fluorescent proteinC2 (pEGFP-C2) was purchased from BD Biosciences
(Franklin Lakes, NJ, USA). The open reading frame encoding TgGRA15 II (omitted signal peptide of $1500 \mathrm{bp}$; http://toxodb.org) cDNA was reconstituted by RT-PCR using total RNA isolated from TgCtwh3 tachyzoites. The pEGFP-GRA15 1 II plasmid was constructed by inserting TgGRA15 ${ }_{\text {II }}$ cDNA into the pEGFP plasmid as previously described [29]. JEG-3 cells were plated in 96-well plates (Corning, Corning, NY, USA) at a density of $10^{4}$ cells/ $\mathrm{ml}$, cultured for $24 \mathrm{~h}$, then transfected with either pEGFP or pEGFP-GRA15 II using Lipofectamine 3000 (Invitrogen, Carlsbad, CA, USA) according to the manufacturer's instructions.

\section{The presence of GRA15 ॥}

The expression of green fluorescent protein (GFP) was recorded using fluorescent microscopy (Olympus BX60, Tokyo, Japan) $24 \mathrm{~h}$ after transfection. The presence of

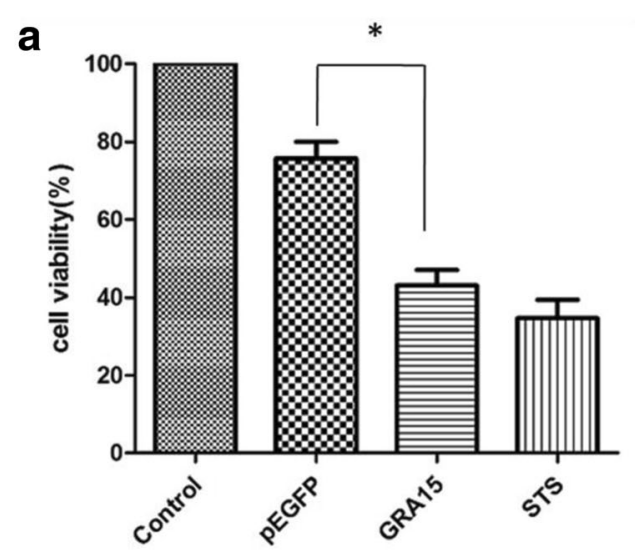

b
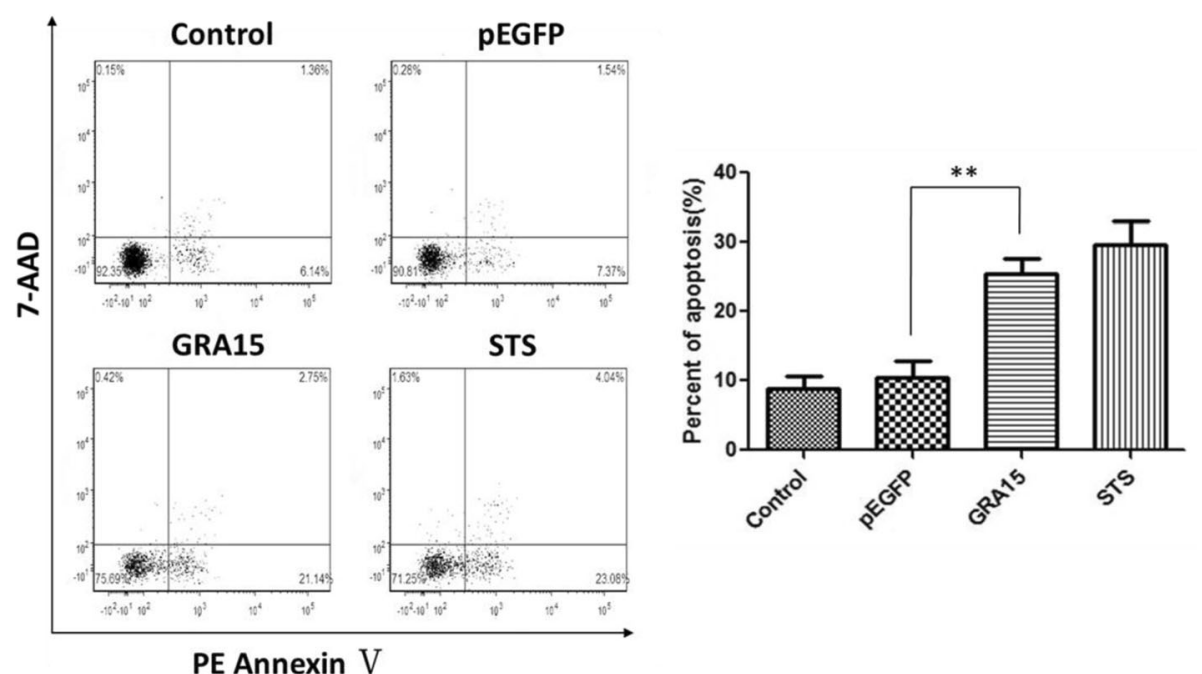

Fig. 2 Dense granule protein 15 (GRA15/)-induced loss of cell viability and apoptosis. Choriocarcinoma JEG-3 cells were transfected with either empty vector (pEGFP, encoding enhanced green fluorescent protein) or pEGFP-GRA15 || for $24 \mathrm{~h}$. Untransfected cells served as the negative control, and staurosporine (STS) treated cells $(1 \mu \mathrm{M}, 6 \mathrm{~h})$ served as the positive control. a Cell viability was measured using the MTS (3-[4,5-dimethylthiazol-2-yl]-5-[3-carboxymethoxyphenyl]-2[4-sulfophenyl]-2H-tetrazolium) assay. $\mathbf{b}$ Cell apoptosis was determined by the phycoerythrin-annexin V/7-AAD flow cytometry assay. ${ }^{*} P<0.05$, ${ }^{* *} P<0.01$ 
GRA15 ${ }_{\text {II }}$ protein in either pEGFP- or pEGFP-GRA15 ${ }_{\text {II }}{ }^{-}$ transfected JEG-3 cells was determined by Western blotting (24 h after transfection). Untransfected JEG-3 cells served as a control.

\section{Cell viability and apoptosis}

Twenty-four hours following transfection, JEG-3 cell viability was measured using the Cell Titer 96 Aqueous One Solution Cell Proliferation assay kit (MTS, Promega, Madison, WI, USA) according to the manufacturer's instructions. A phycoerythrin-annexin $\mathrm{V}$ apoptosis detection kit (flow cytometry based assay, BD Biosciences, Franklin Lakes, NJ,
USA) was used to determine cell apoptosis. Briefly, cells were washed twice using cold phosphate buffered saline (PBS) and suspended in binding buffer $(100 \mu \mathrm{l})$. Phycoerythrin-annexin $\mathrm{V}(5 \mu \mathrm{l})$ and 7-aminoactinomycin $\mathrm{D}(7-\mathrm{AAD}, 5 \mu \mathrm{l})$ were added to the suspension and incubated for $15 \mathrm{~min}$ at room temperature, followed by addition of binding buffer $(400 \mu \mathrm{l})$. A flow cytometry assay was conducted within $1 \mathrm{~h}$ using FACSVerse (BD Biosciences) with FCS Express 4.0. Annexin $\mathrm{V}^{+} / 7-\mathrm{AAD}^{-}$ represented early apoptotic cells and annexin $\mathrm{V}^{+} / 7-\mathrm{AAD}^{+}$ represented late apoptotic cells. Untransfected cells served as a negative control. Cells treated with staurosporine

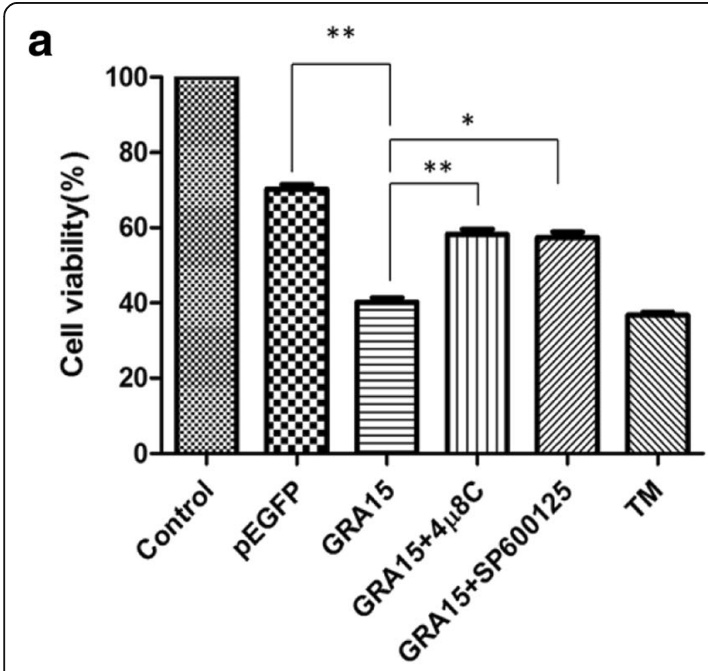

b
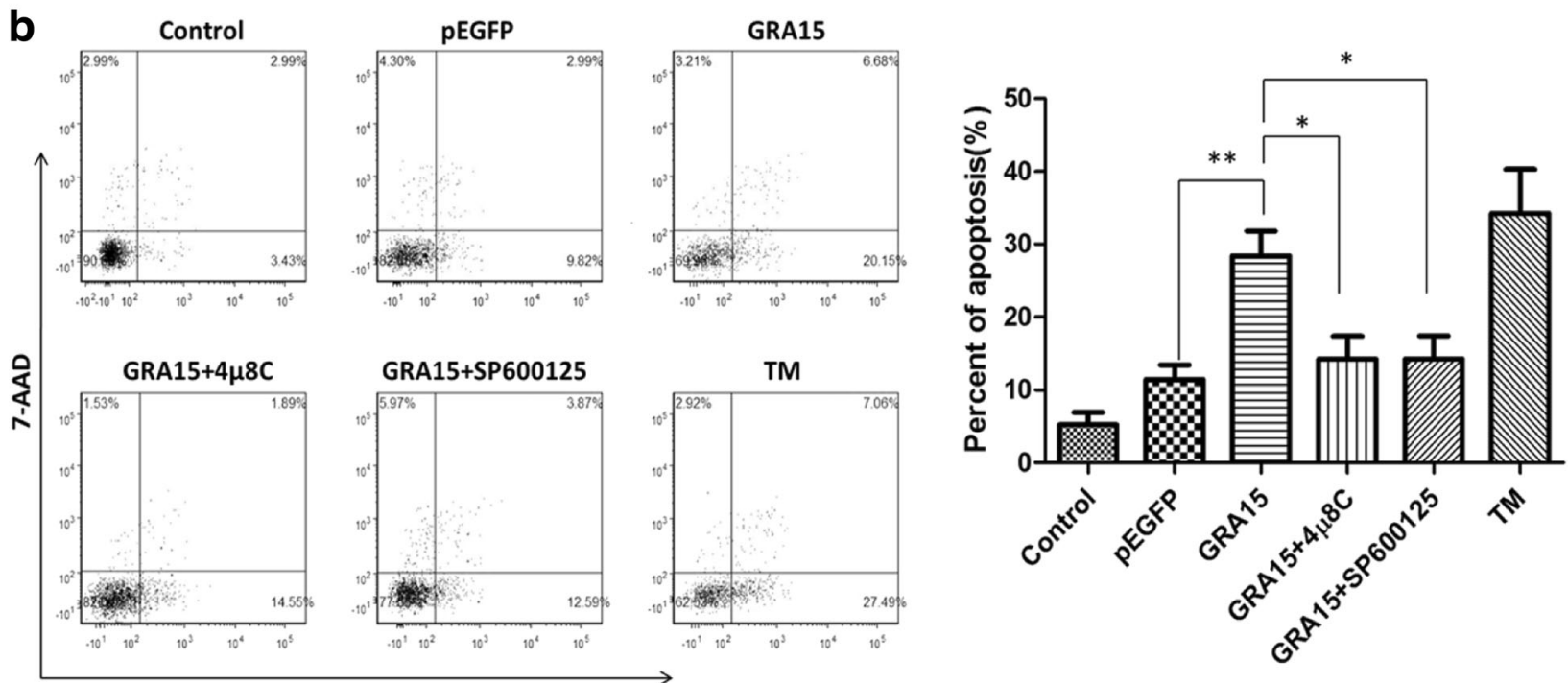

PE Annexin V

Fig. 3 Effects of IRE1a inhibitor 4 48C and JNK inhibitor SP6000125 on loss of cell viability and apoptosis of pEGFP-GRA15,|-transfected cells. Choriocarcinoma JEG-3 cells were transfected with either the empty vector (pEGFP, encoding enhanced green fluorescent protein) or pEGFP-

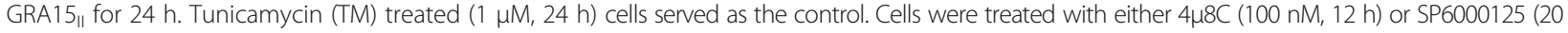
$\mu \mathrm{M}, 12 \mathrm{~h}) 12 \mathrm{~h}$ after pEGFP-GRA15 || transfection. a Cell viability was measured using the MTS (3-[4,5-dimethylthiazol-2-yl]-5-[3-carboxymethoxyphenyl]-2-[4sulfophenyl]-2H-tetrazolium) assay. b Cell apoptosis was determined by the phycoerythrin-annexin V/7-AAD flow cytometry assay. ${ }^{*} P<0.05$, ${ }^{*} P<0.01$ 
(STS, $1 \mu \mathrm{M}, 6 \mathrm{~h}$; Sigma-Aldrich) and/or tunicamycin (TM, $1 \mu \mathrm{M}, 24 \mathrm{~h}$; Sigma) served as positive controls. Cells were also treated with $4 \mu 8 \mathrm{C}$, the inositol-requiring kinase $1 \alpha$ (IRE1 $\alpha$ ) inhibitor (100 $\mathrm{nM}$, added $12 \mathrm{~h}$ after transfection, for $12 \mathrm{~h}$; Selleck, Houston, TX, USA) and/or SP6000125, the c-Jun N-terminal kinase (JNK) inhibitor $(20 \mu \mathrm{M}$, added $12 \mathrm{~h}$ after transfection, for $12 \mathrm{~h}$; Selleck) to demonstrate the effect of GRA15 II on ERS in JEG-3 cells.

\section{Real-time PCR}

Cells were harvested $24 \mathrm{~h}$ after transfection. Total RNAs were extracted, and cDNA was synthesized using the Thermo Fisher Scientific RevertAid First Strand cDNA Synthesis Kit (lot number K1621, Thermo Fisher Scientific, San Diego, CA, USA). Quantitative real-time PCR was performed using the SYBR-Green kit (Takara, Tokyo, Japan) with the ABI7500 system (Applied Biosystems, Carlsbad, CA, USA). Primers listed in Table 1 were synthesized by Shenggong Biotechnology (Shanghai, China). Gene expression levels were normalized to glyceraldehyde-3phosphate dehydrogenase (GAPDH) levels and data was analyzed using the $2^{-\Delta \Delta \mathrm{Ct}}$ method.

\section{Western blotting}

Cells were harvested $24 \mathrm{~h}$ after transfection, washed with cold PBS, and lysed on ice using lysis buffer [50 $\mathrm{mM}$ Tris- $\mathrm{HCl} \mathrm{pH} \mathrm{7.4,} 150 \mathrm{mM} \mathrm{NaCl}, 1 \%$ Triton X$100,1 \%$ sodium deoxycholate, and $0.1 \%$ sodium dodecyl sulfate (SDS), supplemented with protease inhibitors cocktail (1\%) and $1 \mathrm{mM}$ phenylmethanesulfonyl fluoride]. The whole cell lysate was centrifuged $\left(12,000 \times g, 10 \mathrm{~min}, 4{ }^{\circ} \mathrm{C}\right)$, and the supernatant was collected. Protein content was analyzed using a bicinchoninic acid (BCA) assay kit (Beyotime, Shanghai, China). Protein $(20 \mu \mathrm{g})$ was electrophoresed on $10 \%$ SDS-polyacrylamide gels, and transferred to nitrocellulose membranes (Millipore, Billerica, MA, USA). The membranes were blocked using 5\% fat-free milk powder (in PBS) and incubated with primary antibody (1:1000 dilution) for $12 \mathrm{~h}$ at $4{ }^{\circ} \mathrm{C}$. Horseradish peroxidase-conjugated secondary antibody (1:5000 dilution) was then applied at room temperature for 2 h. The membranes were washed and probed using an enhanced chemiluminescence (ECL) kit (Thermo Scientific, Barrington, IL, USA). ImageJ (Version 1.48, National Institute of Health) was used to quantify band density. Antibodies against GFP, C/EBP homologous protein (CHOP), 78-kDa glucose-regulated protein (GRP78), JNK, phospho-JNK, protein kinase R (PKR)-like ER kinase (PERK) and phospho-PERK and GAPDH were purchased from Santa Cruz Biotechnology (Dallas, TX, USA) and were diluted to 1: 1000. Antibodies against cleaved poly (ADP-ribose) polymerase (PARP), cleaved caspase-3, IRE1 $\alpha$, apoptosis signal-regulating kinase 1 (ASK1), phosphoASK1, p38 (a mitogen-activated protein kinase), phospho-p38, X-box binding protein-1 (XBP-1) and TNF receptor-associated factor 2 (TRAF2) were provided by Cell Signaling Technology (Danvers, MA, USA) and were diluted to 1:1000. The antibody to phospho-IRE1 was obtained from Abcam (Cambridge, UK) and was diluted to 1:1000. The antibody to Bax (diluted to 1:1000), and the goat anti-rabbit and goat anti-mouse secondary antibodies (diluted to 1:5000) were purchased from ZSGB-Bio (Beijing, China). Untransfected cells served as a negative control. Cells treated with STS $(1 \mu \mathrm{M}, 6 \mathrm{~h})$ and/or TM $(1 \mu \mathrm{M}, 24 \mathrm{~h})$ served as positive controls. Cells treated with $4 \mu 8 \mathrm{C}$ (100 nM, added $12 \mathrm{~h}$ after transfection, for $12 \mathrm{~h}$ ) and/or SP6000125 $(20 \mu \mathrm{M}$, added $12 \mathrm{~h}$ after transfection, for $12 \mathrm{~h}$ ) were used to demonstrate the effect of GRA15 1 II on ERS in JEG-3 cells.

\section{Statistical analysis}

A two-tailed independent Student's t-test (GraphPad Prism 5.0 software, GraphPad Prism, San Diego, CA, USA) was used to determine the differences between control and pEGFP-GRA15 ${ }_{\mathrm{II}}$-transfected JEG-3 cells. Data are presented as the mean \pm standard error (SE). All statistical tests were considered as significant at $P<0.05$.

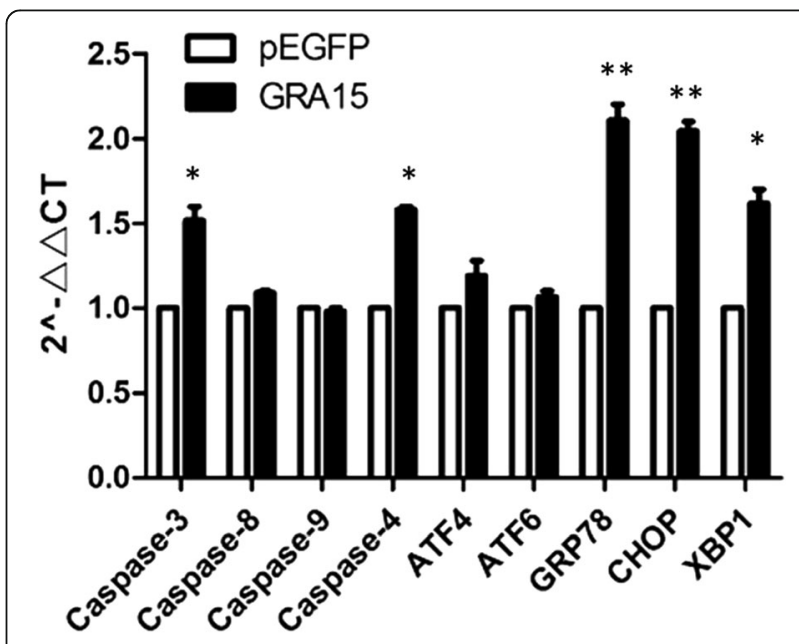

Fig. 4 Transcription levels of apoptosis-associated genes. Choriocarcinoma JEG-3 cells were transfected with either the empty vector (pEGFP, encoding enhanced green fluorescent protein) or pEGFP-GRA15 „॥ for 24 h. Abbreviations: ATF, activating transcription factor; GRP78, 78kDa glucose-regulated protein; $\mathrm{CHOP}, \mathrm{C} / \mathrm{EBP}$ homologous protein; XBP1, X-box binding protein-1. ${ }^{*} P<0.05$, ${ }^{*} P<0.01$, when compared to the pEGFP-transfected cells 


\section{Results}

The presence of GRA15 ॥

To investigate whether the pEGFP-GRA15 1 II construct could be expressed in JEG-3 cells, the expression of GRA15 II protein was determined in both pEGFPGRA15 II- $^{-}$and pEGFP-transfected JEG-3 cells. GFP fluorescence was detected in both pEGFP and pEGFPGRA15 II -transfected JEG-3 cells at $24 \mathrm{~h}$ (Fig. 1a). pEGFP-transfected cells had an increased fluorescence signal when compared to pEGFP-GRA15 $5_{\text {II }}$-transfected cells. The GFP protein $(28 \mathrm{kDa})$ and the GFP-GRA15 II fusion protein $(85 \mathrm{kDa})$ were expressed in JEG-3 cells, $24 \mathrm{~h}$ after transfection (Fig. 1b).
Loss of cell viability and apoptosis

Viability and apoptosis, that may directly reflect the functional status of the cells, were analyzed in both pEGFP-GRA15 II- $^{-}$and pEGFP-transfected JEG-3 cells. Untransfected JEG-3 cells exhibited 100\% viability and 7 . 5\% apoptosis (Fig. 2). STS treatment decreased cell viability to $39.4 \%$ and increased apoptosis to $27.8 \%$. The pEGFP-GRA15 II $_{\text {-transfected cells showed decreased cell }}$ viability $\left(t_{(2)}=5.611, P=0.0303,47.0 v s 74.5 \%\right)$ and increased cell apoptosis $\left(t_{(2)}=10.74, P=0.0086,23.9\right.$ vs 8 . 9\%) when compared to pEGFP-transfected cells. Interestingly, while pEGFP-GRA15 II transfection decreased viability $\left(t_{(2)}=10.23, P=0.0094\right)$ and increased

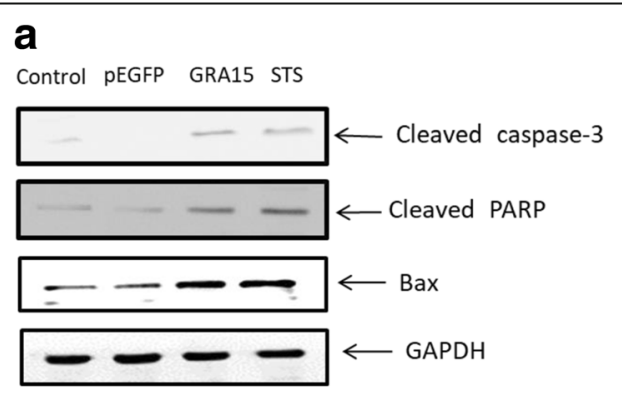

b
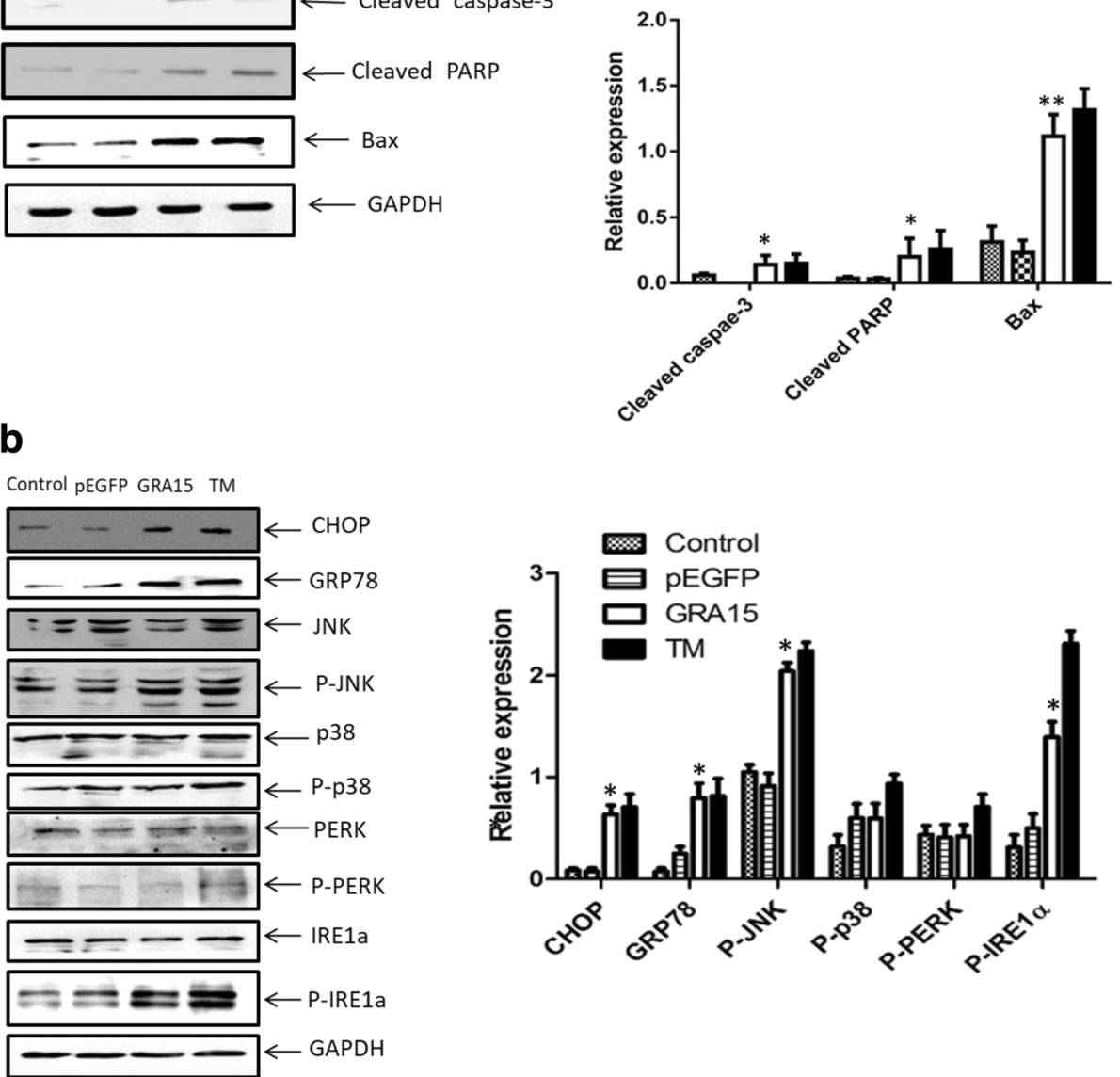

Fig. 5 Expression of apoptosis-associated proteins and endoplasmic reticulum stress (ERS) proteins. Choriocarcinoma JEG-3 cells were transfected with either the empty vector (pEGFP, encoding enhanced green fluorescent protein) or pEGFP-GRA15 for 24 h. Untransfected cells served as the negative control. a Expression of apoptosis-associated proteins. Staurosporine (STS) treated cells ( $1 \mu \mathrm{M}, 6 \mathrm{~h})$ served as the positive control. $\mathbf{b}$ Expression of ERS proteins. Tunicamycin (TM) treated cells (1 $\mathrm{MM}, 24 \mathrm{~h}$ ) served as the positive control. Abbreviations: PARP, poly (ADP-ribose) polymerase; GAPDH, glyceraldehyde-3-phosphate dehydrogenase; CHOP, C/EBP homologous protein; GRP78, 78-kDa glucose-regulated protein; JNK, cJun N-terminal kinase; P-JNK, phosphorylated JNK; P38, a mitogen-activated protein kinase; P-p38, phosphorylated p38; PERK, PRK-like ER kinase; PPERK, phosphorylated PERK; IRE1a, inositol requiring kinase 1; P-IRE1a, phosphorylated IRE1a. * $P<0.05$, **P<0.01, when compared to the pEGFP-transfected cells 
apoptosis $\left(t_{(2)}=4.851, P=0.04\right)$ of JEG-3 cells, treatment with either $4 \mu 8 \mathrm{C}$ (IRE1 $\alpha$ inhibitor) or SP6000125 (JNK inhibitor) increased viability $\left(t_{(2)}=9,165, P=0\right.$. $0117)$ and decreased apoptosis $\left(t_{(2)}=6,963, P=0.02\right)$ in

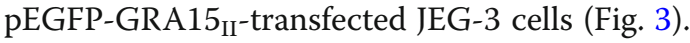

\section{Transcription levels of apoptosis-associated genes and \\ ERS genes}

The transcription levels of apoptosis-associated genes and ERS genes were measured in both pEGFPGRA $15_{\mathrm{II}^{-}}$and pEGFP-transfected JEG-3 cells so as to reveal the signal pathway of the cell apoptosis. The pEGFP-GRA15 ${ }_{\text {II }}$-transfected JEG-3 cells demonstrated increased mRNA expression levels of caspase-3 $\left(t_{(2)}=\right.$ 6.229, $P=0.0248)$, caspase $-4\left(t_{(2)}=5.819, P=0.0283\right)$, GRP78 $\left(t_{(2)}=11.632, P=0.0073\right)$, CHOP $\left(t_{(2)}=18.298\right.$, $P=0.003)$ and XBP1 $\left(t_{(2)}=7.589, P=0.0169\right)$ when compared to pEGFP-transfected JEG-3 cells (Fig. 4).

\section{Expression of apoptosis-associated proteins and ERS proteins}

Accordingly, the expression levels of apoptosis-associated proteins and ERS proteins were measured in both pEGFP-
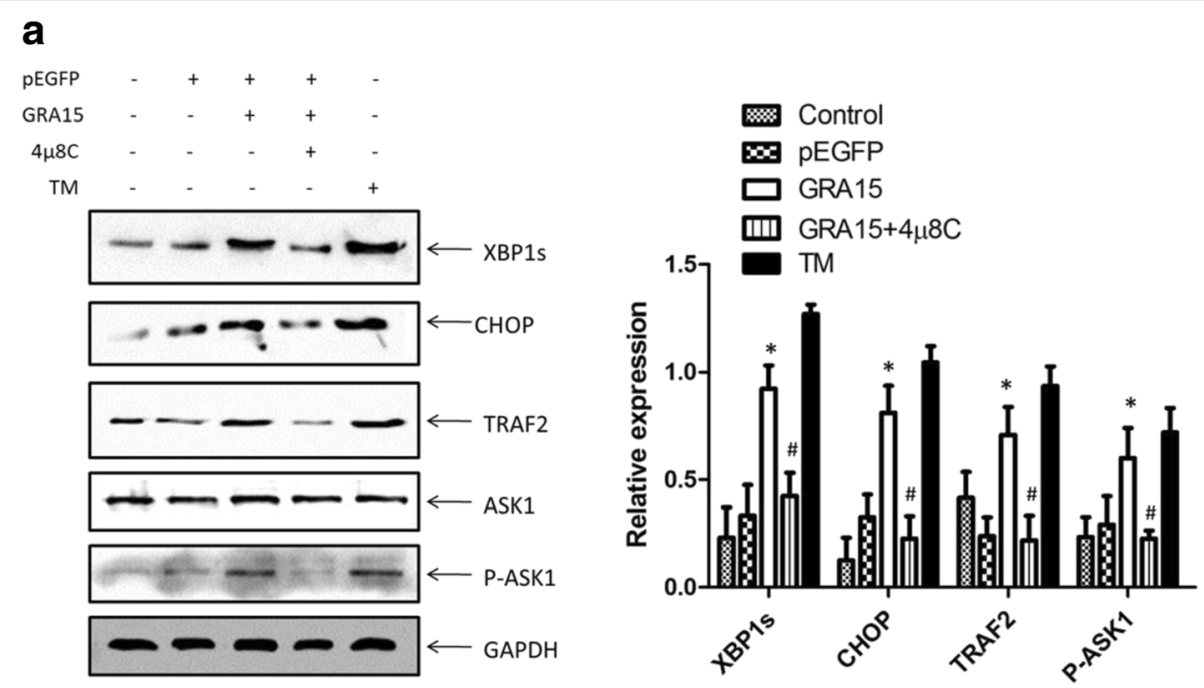

b
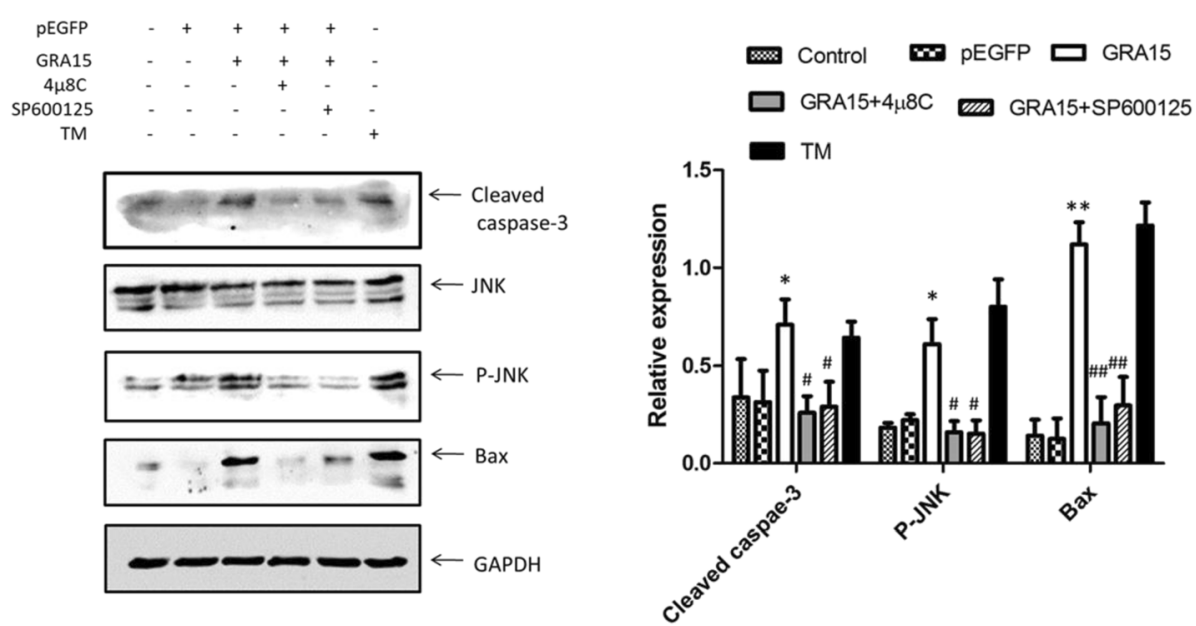

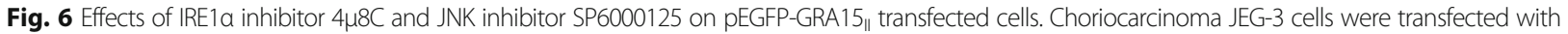

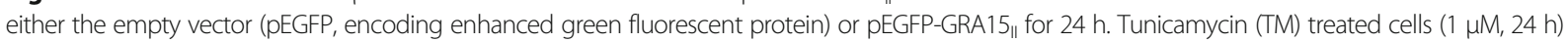
served as a control. a Cells were treated with $4 \mu 8 \mathrm{C}(100 \mathrm{nM}, 12 \mathrm{~h}) 12 \mathrm{~h}$ after pEGFP-GRA15 t transfection. ${ }^{*} P<0.05$, when compared to pEGFP transfected

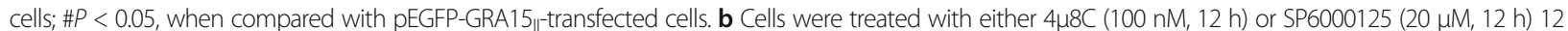
h after pEGFP-GRA15 | transfection. ${ }^{*} P<0.05$, ${ }^{* *} P<0.01$, when compared to pEGFP transfected cells; $\# P<0.05$, \#\#P<0.01, when compared to pEGFPGRA15 „-transfected cells. Abbreviations: XBP1, X-box binding protein-1; CHOP, C/EBP homologous protein; TRAF2, TNF receptor-associated factor 2; ASK1, apoptosis signal-regulating kinase 1; P-ASK1, phosphorylated ASK1; GAPDH, glyceraldehyde-3-phosphate dehydrogenase; JNK, c-Jun N-terminal kinase; PJNK, phosphorylated JNK 
GRA15 ${ }_{\text {II }}$ - and pEGFP-transfected JEG-3 cells. When compared to pEGFP-transfected cells, the pEGFP-GRA15 transfected cells showed increased expression levels of apoptosis-associated proteins (Fig. 5a) such as cleaved caspase-3 $\left(t_{(2)}=4.797, P=0.0408\right)$, cleaved PARP $\left(t_{(2)}=4\right.$. $728, P=0.0419)$ and $\operatorname{Bax}\left(t_{(2)}=24.489, P=0.0017\right)$, as well as increased expression levels of ERS proteins (Fig. 5b), such as CHOP, GRP78, phospho-JNK and phospho-IRE1 $\alpha$ (all $P<0.05$ ). Interestingly, $4 \mu 8 \mathrm{C}$ treatment decreased the expression levels of XBP1s, CHOP, TRAF2 and phosphoASK1 in pEGFP-GRA15 1 -transfected JEG-3 cells (all $P<0$. 05 , Fig. 6a). In addition, treatment with either $4 \mu 8 \mathrm{C}$ or SP6000125 decreased the expression levels of cleaved caspase-3, phospho-JNK, and Bax in pEGFP-GRA15 ${ }_{\mathrm{II}^{-}}$ transfected JEG-3 cells (all $P<0.05$, Fig. 6b).

\section{Discussion}

Toxoplasma gondii infection can cause abortion, preterm delivery, stillbirth and fetal abnormalities in pregnant animals and humans $[30,31]$ through three possible mechanisms. First, Toxoplasma gondii can be directly transferred to the fetus through the placenta and cause a congenital infection [25]; secondly, maternal physiological and immunological disorders caused by Toxoplasma gondii infection can adversely affect fetal development [26]; and thirdly, Toxoplasma gondii can induce apoptosis in placental cells [27]. It has been found that the majority of host cells act as bystanders during an acute infection, and apoptosis of these host cells may result from the secretion of certain soluble factors by parasite-infected cells [32, 33]. The composition of the T. gondii excreted-secreted antigens (ESAs) is surprisingly complex and only a few microneme proteins, ROPs and GRAs, have been identified [34, 35]. ROP16- and ROP18-induced host cell apoptosis has been previously reported $[21,22]$. In this study, GRA15 II transfection significantly increased apoptosis and decreased cell viability in choriocarcinoma JEG-3 cells as early as 24 hours post-transfection. These results suggest that GRA15 1 , which exists in Toxoplasma gondii strains such as ME49 (type II Toxoplasma gondii) and ToxoDB\#9 (major Toxoplasma gondii strain in China), is a virulence antigen.

The GRA15 ${ }_{\text {II }}$-induced apoptosis was accompanied with ERS in choriocarcinoma JEG-3 cells. The mRNA transcription and protein expression level of GRP78, a key ERS sensor protein, were significantly increased by GRA15 II transfection at 24 hours. The finding indicates that GRA15 1 -induced apoptosis at least partially resulted from ERS. In other studies, PERK, activating transcription factor (ATF) 6, and IRE1 $\alpha$ have been proposed as three major proteins downstream of GRP78 signaling during ERS [36, 37]. In the current study, the expression level of phospho-IRE1 $\alpha$, but not PERK or ATF6, was increased by GRA15 1 Ir transfection. In line with this, the GRA15 1 -related ERS/apoptosis was mainly induced by the GRP78-IRE1 $\alpha$ signaling pathway. It has been reported that the activation (phosphorylation) of IRE1 $\alpha$ could lead to apoptosis by either mediating the splicing of XBP1 to XBP1s, and subsequently increasing the expression of CHOP [38], or recruiting TRAF2, activating ASK1, and stimulating JNK [39]. In the current work, we found the expression of proteins in both of these pathways was significantly increased by GRA15 ${ }_{\text {II }}$ transfection. Thus, the result clearly demonstrates that both the IRE1 $\alpha$-XBP1-CHOP and IREl $\alpha$ TRAF2-ASK1-JNK pathways contributed in GRA15 $\mathrm{II}^{-}$ induced apoptosis as proposed schematically in Fig. 7. We further illustrated this hypothesis by treating the GRA15 II-transfected choriocarcinoma JEG-3 cells with $4 \mu 8 \mathrm{C}$ (IREl $\alpha$ inhibitor) and SP6000125 (JNK inhibitor); both $4 \mu 8 \mathrm{C}$ and SP6000125 suppressed the expression levels of apoptosis-associated proteins in GRA15 ${ }_{\mathrm{II}}$-transfected choriocarcinoma JEG-3 cells, and subsequently decreased apoptosis and increased cell viability.

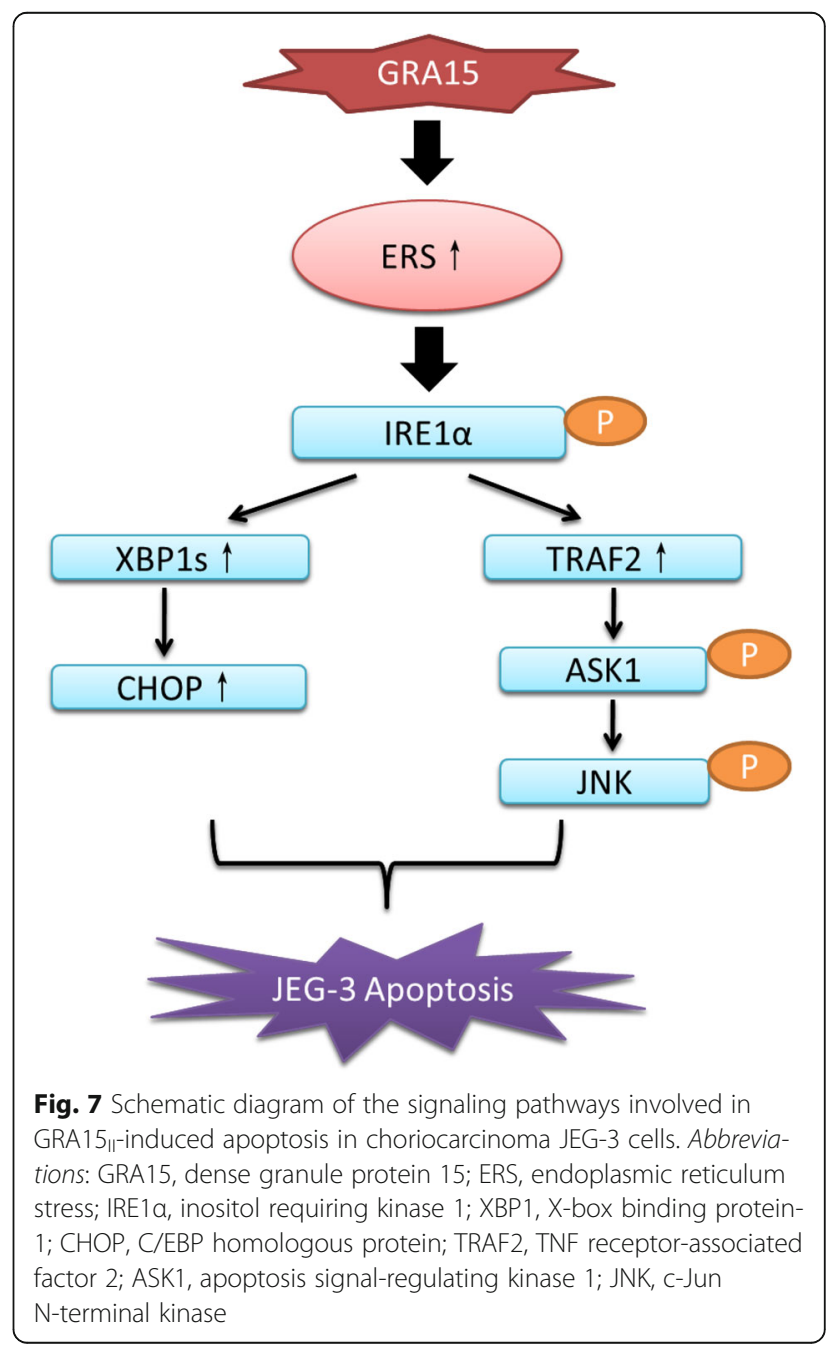


During pregnancy, fetal development is directly related to the proliferation, differentiation, and apoptosis of trophoblast cells [23, 24]. Increased trophoblast cell apoptosis could be damaging to fetal health and even cause adverse pregnancy outcomes [40, 41]. The above finding contributes novel knowledge to our current understanding in regards to Toxoplasma gondii-induced apoptosis, and may help to illustrate the underlying mechanism of Toxoplasma gondii-induced pregnancy failure. The objective of the current study was to determine whether ERS was involved in GRA15 1 -induced apoptosis in choriocarcinoma JEG-3 cells. It was not investigated whether ERS was the only (or the major) factor that caused apoptosis in GRA15 $5_{\mathrm{II}}$-transfected choriocarcinoma JEG-3 cells. It is possible that other pathways (e.g. mitochondrial pathway, death receptor pathway) may also contribute to GRA15 ${ }_{\mathrm{II}}$-induced cell apoptosis, and this will need to be investigated in future studies. Additionally, the host target protein of GRA15 II remains unknown. There may be some differences in "normal" in vivo cells when compared to the choriocarcinoma JEG-3 cells that were used in the current study.

\section{Conclusions}

Toxoplasma-derived GRA15 1 in increased the expression of ERS- and apoptosis-associated proteins in choriocarcinoma JEG-3 cells. GRA15 ${ }_{\text {II }}$-induced ERS and apoptosis were alleviated by treatment with $4 \mu 8 \mathrm{C}$ and SP6000125. GRA15 II induces apoptosis at least partially through endoplasmic reticulum stress.

\section{Abbreviations \\ GRA15: dense granule protein 15; ROP16: rhoptry protein 16; ERS: endoplasmic reticulum stress; STS: staurosporine; TM: tunicamycin; ATF: activating transcription factor; GRP78: 78-kDa glucose-regulated protein; CHOP: C/EBP homologous protein; XBP1: X-box binding protein-1; PARP: poly (ADP-ribose) polymerase; JNK: c-Jun N-terminal kinase; GAPDH: glyceraldehyde-3-phosphate dehydrogenase; P-JNK: phosphorylated JNK; P38: a mitogen-activated protein kinase; P-p38: phosphorylated p38; PERK: PRK-like ER kinase; P-PERK: phosphorylated PERK; IRE1a: inositol requiring kinase 1; P-IRE1a: phosphorylated IRE1a; TRAF2: TNF receptor- associated factor 2; ASK1: apoptosis signal-regulating kinase 1; P- ASK1: phosphorylated ASK1}

\section{Acknowledgements}

The authors thank Dexiang Xu, Deyong Chu, Jing Chao, Min Li and Weisheng Cheng for their kind support.

\section{Funding}

This study was financially supported by the National Natural Science Foundation of China (Grant No. 81471983; and No. 81572022).

\section{Availability of data and materials}

The data supporting the conclusions of this article are included within the article. The datasets generated and analyzed during the current study are available from the corresponding author upon reasonable request.

\section{Authors' contributions}

JLS, LIZ and WW elaborated and designed the study. WW, FFZ and HC performed the experiments and analyzed the data. WW and JLS drafted the manuscript. YYT, TX, QLL, LY and JD participated in the analysis and interpretation of data. All authors read and approved the final manuscript.

\section{Ethics approval and consent to participate}

All of the protocols used in the study were approved by the Institutional Review Board of the Institute of Biomedicine at Anhui Medical University, Hefei, Anhui, China (Permit Number AMU26-080610).

\section{Competing interests}

The authors declare that they have no competing interests.

\section{Author details}

${ }^{1}$ Department of Immunology, School of Basic Medicine, Anhui Medical University, Hefei 230032, China. ${ }^{2}$ Department of Pathogen Biology and the Key Laboratory of Microbiology (Anhui), School of Basic Medicine, Anhui Medical University, Hefei 230032, China. ${ }^{3}$ Laboratory of Clinical Diagnostics, the First Hospital of Anhui Medical University, Hefei 230032, China. ${ }^{4}$ Key Laboratory of Oral Diseases Research of Anhui Province, Hospital of Stomatology, Anhui Medical University, Hefei 230032, China.

Received: 10 January 2018 Accepted: 6 April 2018

Published online: 17 April 2018

\section{References}

1. Dubremetz JF. Host cell invasion by Toxoplasma gondii. Trends Microbiol. 1998;6:27-30.

2. Howe DK, Sibley LD. Toxoplasma gondii comprises three clonal lineages: correlation of parasite genotype with human disease. J Infect Dis. 1995; 172(6):1561

3. Howe DK, Honore S, Derouin F. Determination of genotypes of Toxoplasma gondii strains isolated from patients with toxoplasmosis. J Clin Microbiol. 1997:35:1411-4.

4. Dardé ML, Bouteille B, Pestre-Alexandre M. Isoenzyme analysis of 35 Toxoplasma gondii isolates and the biological and epidemiological implications. J Parasitol. 1992;78:786-94.

5. Ajzenberg D, Bañuls AL, Tibayrenc M, Dardé ML. Microsatellite analysis of Toxoplasma gondii shows considerable polymorphism structured into two main clonal groups. Int J Parasitol. 2002;32:27-38.

6. Saeij JPJ, Coller S, Boyle JP, Jerome ME, White MW, Boothroy JC. Toxoplasma co-opts host gene expression by injection of a polymorphic kinase homologue. Nature. 2007:445:324-7.

7. Laliberte J, Carruthers V. Host cell manipulation by the human pathogen Toxoplasma gondii. Cell Mol Life Sci. 2008:65:1900-15.

8. Hunter CA, Sibley LD. Modulation of innate immunity by Toxoplasma gondii virulence effectors. Nat Rev Microbiol. 2012;10:766-78.

9. Murray PJ. Macrophages as a battleground for Toxoplasma pathogenesis. Cell Host Microbe. 2011;9:445-7.

10. Zhou P, Sun XT, Yin CC, Yang JF, Yuan ZG, Yan HK, et al. Genetic characterization of Toxoplasma gondii isolates from pigs in southwestern China. J Parasitol. 2011:96:1193-5.

11. Chen ZW, Gao JM, Huo XX, Wang L, Yu L, Halmlai F, et al. Genotyping of Toxoplasma gondii isolates from cats in different geographic regions of China. Vet Parasitol. 2011;183:166-70.

12. Cai $Y$, Chen H, Mo X, Tang Y, Xu X, Zhang A, et al. Toxoplasma gondii inhibits apoptosis via a novel STAT3-miR-17-92-Bim pathway in macrophages. Cell Signal. 2014;26:1204-12.

13. Cheng W, Liu F, Li M, Hu X, Chen H, Pappoe F, et al. Variation detection based on next-generation sequencing of type Chinese 1 strains of Toxoplasma gondii with different virulence from China. BMC Genomics. 2015;16:888

14. Cheng W, Wang C, Xu T, Liu F, Pappoe F, Luo Q, et al. Genotyping of polymorphic effectors of Toxoplasma gondii isolates from China. Parasit Vectors. 2017;10:580

15. Contreras-Ochoa CO, Lagunas-Martínez A, Belkind-Gerson J, Díaz-Chávez J, Correa D. Toxoplasma gondii invasion and replication within neonate mouse astrocytes and changes in apoptosis related molecules. Exp Parasitol. 2013; 134:256-65.

16. Gavrilescu LC, Denkers EY. IFN-overproduction and high level apoptosis are associated with high but not low virulence Toxoplasma gondii infection. J Immunol. 2001;167:902-9.

17. El-Sagaff S, Salem HS, Nichols W, Tonkel AK, Abo-Zenadah NY. Cell death pattern in cerebellum neurons infected with Toxoplasma gondii. J Egypt Soc Parasitol. 2005;35:809-18. 
18. Angeloni MB, Guirelli PM, Franco PS, Barbosa BF, Gomes AO, Castro AS, et al. Differential apoptosis in BeWo cells after infection with highly $(\mathrm{RH})$ or moderately (ME49) virulent strains of Toxoplasma gondii is related to the cytokine profile secreted, the death receptor Fas expression and phosphorylated ERK1/2 expression. Placenta. 2013;34:973-82.

19. Wang T, Zhou J, Gan X, Wang H, Ding X, Chen L, et al. Toxoplasma gondii induces apoptosis of neural stem cells via endoplasmic reticulum stress pathway. Parasitology. 2014;141:988-95.

20. Zhou J, Gan X, Wang Y, Zhang X, Ding X, Chen L, et al. Toxoplasma gondii prevalent in China induce weaker apoptosis of neural stem cells C17.2 via endoplasmic reticulum stress (ERS) signaling pathways. Parasit Vectors. 2015;8:73.

21. Chang S, Shan X, Li X, Fan W, Zhang SQ, Zhang J, et al. Toxoplasma gondii rhoptry protein ROP16 mediates partially SH-SY5Y cells apoptosis and cell cycle arrest by directing ser15/37 phosphorylation of p53. Int J Biol Sci. 2015;11:1215-25.

22. Wan L, Gong L, Wang W, An R, Zheng M, Jiang Z, et al. T. gondii rhoptry protein ROP18 induces apoptosis of neural cells via endoplasmic reticulum stress pathway. Parasit Vectors. 2015;8:554.

23. Soni S, Rath G, Prasad CP, Salhan S, Saxena S, Apoptosis JAK. BCl-2 protein expression in human placenta over the course of normal pregnancy. Anat Histol Embryol. 2010;39:426-31.

24. De FM, Penta R, Laforgia V, Cobellis L, De LA. Apoptosis and human placenta: expression of proteins belonging to different apoptotic pathways during pregnancy. J Exp Clin Cancer Res Cr. 2005;24:25-33.

25. Pappas G, Roussos N, Falagas ME. Toxoplasmosis snapshots: global status of Toxoplasma gondii seroprevalence and implications for pregnancy and congenital toxoplasmosis. Int J Parasitol. 2009;39:1385-94.

26. Han M, Jiang Y, Lao K, Xu X, Zhan S, Wang Y, et al. sHLA-G involved in the apoptosis of decidual natural killer cells following Toxoplasma gondii infection. Inflammation. 2014;37:1718-27.

27. Liu T, Zhang Q, Liu L, Xu X, Chen H, Wang H, et al. Trophoblast apoptosis through polarization of macrophages induced by Chinese Toxoplasma gondii isolates with different virulence in pregnant mice. Parasitol Res. 2013; 112:3019-27.

28. Rosowski EE, Lu D, Julien L, Rodda L, Gaiser RA, Jensen KDC, et al. Strainspecific activation of the NF-kB pathway by GRA15, a novel Toxoplasma gondii dense granule protein. J Exp Med. 2011;208:195-212.

29. Xie $Y$, Wen $H$, Yan $K$, Wang $S$, Wang $X$, Chen J, et al. Toxoplasma gondii GRA15II effector-induced M1 cells ameliorate liver fibrosis in mice infected with schistosomiasis japonica. Cell Mol Immunol. 2018;15(2):120-34.

30. Kravetz JD, Federman DG. Toxoplasmosis in pregnancy. Am J Med. 2005; 118:212-6.

31. Petersen E. Prevention and treatment of congenital toxoplasmosis. Expert Rev Anti Infect Ther. 2007:5:285-93.

32. Nishikawa $Y$, Kawase $O$, Vielemeyer $O$, Suzuki $H$, Joiner KA, Xuan $X$, et al. Toxoplasma gondii infection induces apoptosis in noninfected macrophages: role of nitric oxide and other soluble factors. Parasite Immunol. 2007:29:375-85.

33. Mordue DG, Monroy F, La Regina M, Dinarello CA, Sibley LD. Acute toxoplasmosis leads to lethal overproduction of Th1 cytokines. J Immunol. 2001;167:4574-84.

34. Gladkova SE, Bormotov NI, Dedkova LM, Reshetnikov SS, Kurlaeva TB, Belanov EF. An immunochemical study of the antigens from Toxoplasma gondii tachyzoites obtained in different cultivation systems. Med Parazitol (Mosk). 1998;1:20-3.

35. Zhou XW, Kafsack BFC, Cole RN, Beckett P, Shen RF, Carruthers VB. The opportunistic pathogen Toxoplasma gondii deploys a diverse legion of invasion and survival proteins. J Biol Chem. 2005;280:34233-44.

36. Hossain MM, Richardson JR. Mechanism of pyrethroid pesticide-induced apoptosis: Role of calpain and the ER stress pathway. Toxicol Sci. 2011; 122:512-25.

37. Hetz C. The unfolded protein response: Controlling cell fate decisions under ER stress and beyond. Nat Rev Mol Cell Biol. 2012;13:89-102.

38. Cadena SG, Hernández-Fonseca K, Camacho-Arroyo I, Massieu L. Glucose deprivation induces reticulum stress by the PERK pathway and caspase-7and calpain-mediated caspase-12 activation. Apoptosis. 2014;19:414-27.

39. Urano F, Wang XZ, Bertolotti A, Zhang Y, Chung P, Harding HP, et al. Coupling of stress in the endoplasmic reticulum to activation of JNK protein kinases by transmembrane protein Kknase IRE1. Science. 2000;287:664-6.
40. Chen SJ, Liu YL, Sytwu HK. Immunologic regulation in pregnancy: from mechanism to therapeutic strategy for immunomodulation. Clin Dev Immunol. 2012; https://doi.org/10.1155/2012/258391.

41. Li CF, Gou WL, Li XL, Wang SL, Yang T, Chen Q. Reduced expression of survivin, the inhibitor of apoptosis protein correlates with severity of preeclampsia. Placenta. 2012;33:47-51.

\section{Ready to submit your research? Choose BMC and benefit from:}

- fast, convenient online submission

- thorough peer review by experienced researchers in your field

- rapid publication on acceptance

- support for research data, including large and complex data types

- gold Open Access which fosters wider collaboration and increased citations

- maximum visibility for your research: over $100 \mathrm{M}$ website views per year

At BMC, research is always in progress.

Learn more biomedcentral.com/submissions 